\title{
What is the best tool for rescue?
}

\author{
Jonathan W. Haft, MD
}

\author{
From the Department of Cardiac Surgery, University of Michigan, Ann Arbor, Mich \\ Disclosures: Author has nothing to disclose with regard to commercial support. \\ Received for publication Nov 26, 2017; accepted for publication Dec 4, 2017. \\ Address for reprints: Jonathan W. Haft, MD, Department of Cardiac Surgery, University of Michigan, 1500 E. \\ Medical Center Dr, Ann Arbor, MI 48109 (E-mail: haft@umich.edu). \\ J Thorac Cardiovasc Surg 2018;155:1069-70 \\ $0022-5223 / \$ 36.00$ \\ Copyright (c) 2017 by The American Association for Thoracic Surgery \\ https://doi.org/10.1016/j.jtcvs.2017.12.032
}

Durable ventricular assist devices (VADs) are an important treatment for end-stage heart failure. Advances in technology have improved outcomes and prolonged survival. However, adverse events continue to persist, affecting mortality and the high frequency of readmissions. Durable VAD therapy is expensive and consumes substantial health care resources, including the primary implant hospitalization and throughout the remaining lifetime of the patient. ${ }^{1}$ As such, the VAD community must serve as good stewards of the technology, applying therapy to those most likely to sustainably benefit. Patients are carefully selected by multidisciplinary committees and meticulously optimized to improve chances of success.

In this issue of the Journal, Pawale and colleagues ${ }^{2}$ report their experience with emergency durable VAD implantation for patients in critical cardiogenic shock. Over a 9-year period, they identified 43 patients considered to be in refractory cardiogenic shock who underwent primary implantation of a durable VAD. Approximately half of these patients were diagnosed with acute myocardial infarctions, and the remainder were thought to be in decompensated chronic heart failure. Early perioperative mortality was $14 \%$, and survival to 6 months and 1 year were $83 \%$ and $74 \%$, respectively. The authors should be congratulated for their excellent outcomes in an extremely ill cohort of patients.

Published evidence to date clearly demonstrates worse survival after VAD implantation for patients in critical cardiogenic shock, the so-called Interagency Registry for Mechanically Assisted Circulatory Support (INTERMACS) class 1 population. ${ }^{3}$ Many centers increasingly prefer to avoid urgent implantation in this setting, rather attempting to optimize these patients pharmacologically or with the use of temporary mechanical circulatory support (TMCS), allowing a period of stabilization and resolution of end organ injury. This also permits a suitable time interval to assess the patients' candidacy for VAD, encompassing the potential medical, social, and psychologic contraindications. There has been a significant decline in the frequency of INTERMACS 1 and 2 durable VAD use compared with the lower acuity categories.

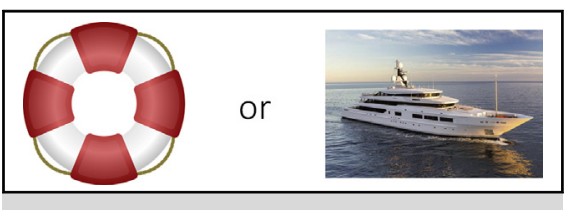

What is the best tool for rescue?

Central Message

Both temporary and durable mechanical circulatory support can restore circulation and improve survival for patients in refractory cardiogenic shock. Which strategy is best? The debate goes on.

See Article page 1059.

Has this report provided enough evidence to influence current practice, encouraging centers to adopt a more aggressive strategy of emergency VAD implantation in patients with cardiogenic shock? In my opinion, it has not. Their intermediate survival was substantially lower than results from the Advance clinical trial in transplant-eligible patients $(94 \%$ and $86 \%$ at 180 and 360 days, respectively) ${ }^{4}$ and the 6-month survival in the Momentum 3 trial comparing the HeartMate 3 (Thoratec Corp, Pleasanton, Calif) with the HeartMate II (Thoratec Corp) in transplant-eligible patients $(91.4 \%$ and $90.1 \%$, respectively). ${ }^{5}$ Furthermore, the authors did not fully report the frequency of adverse events as defined by INTERMACS. To comprehensively understand the potential impact of VAD implantation for a sicker population of patients, we must accurately characterize not only mortality but also the frequency of rehospitalization for events like stroke, malignant dysrhythmias, right ventricular failure, and bleeding. In addition, there was presumably some element of selection bias in their reporting. The authors have not provided information about the other patients presenting in cardiogenic shock at their institution during the same time interval who did not undergo emergency VAD implant. Were some of those patients stabilized and did they undergo VAD implant at a later time point? What were their outcomes? Some patients in their series were in fact on TMCS as a bridge to durable VAD. Why was TMCS chosen in those cases? Last, several patients perhaps should not have received durable VADs at all because of advanced malignancy, social contraindications, or inadequate time to allow for myocardial recovery. 
As a strong proponent for VADs in advanced heart failure, I would like to see expanded use of this rewarding therapy. Although I am impressed with the results reported by Pawale and colleagues, ${ }^{2}$ I remain skeptical that durable VADs are the ideal strategy for critical cardiogenic shock with end-organ injury. Many in the VAD community hope to have the opportunity to evaluate the potential role of mechanical circulatory support for patients with lessadvanced heart failure. This will only be possible when marked improvements in survival and the frequency of adverse events are demonstrated. To achieve this goal, we must be as fastidious with patient selection as we are with surgical techniques and postoperative management.

\section{References}

1. Nunes AJ, MacArthur RG, Kim D, Singh G, Buchholz H, Chatterley P, et al. A systematic review of the cost-effectiveness of long-term mechanical circulatory support. Value Health. 2016;19:494-504.

2. Pawale A, Schwartz Y, Shinobu I, Pinney S, Adams DH, Anyanwu AC Selective implantation of durable left ventricular assist devices as primary therapy for refractory cardiogenic shock. J Thorac Cardiovasc Surg. 2018;155: 1059-68.

3. Kirklin JK, Naftel DC, Pagani FD, Kormos RL, Stevenson LW, Blume ED, et al. Seventh INTERMACS annual report: 15,000 patients and counting. J Heart Lung Transplant. 2015;34:1495-504.

4. Aaronson KD, Slaughter MS, Miller LW, McGee EC, Cotts WG, Acker MA, et al. Use of an intrapericardial, continuous-flow, centrifugal pump in patients awaiting heart transplantation. Circulation. 2012;125:3191-200.

5. Rogers JG, Pagani FD, Tatooles AJ, Bhat G, Slaughter MS, Birks EJ, et al. Intrapericardial left ventricular assist device for advanced heart failure. $N$ Engl J Med. 2017;376:451-60. 